Aim of the study: Metaplastic breast carcinomas (MBC) are a rare group of cancers, accounting for about $1 \%$ of all breast cancers. The study presents a case series of MBC patients diagnosed, treated and followed up in one healthcare center.

Material and methods: The study group comprised 18 women at the median age of 63 years. The most common carcinoma type in the study group was MBC with squamous epithelial differentiation (56\%). Estrogen receptor expression was identified in one patient. No steroid or HER2 receptor expression was found in the remaining patients. We analyzed recurrence and survival rates in relation to clinical and therapeutic factors by using the Kaplan-Meier method.

Results: A significantly longer overall survival time was noted among patients treated with adjuvant radiation therapy, $p=0.018$. No other factors had a significant influence on survival. Because of the small size of the study group, results obtained in the study should be treated with caution.

Key words: metaplastic breast cancer, radiotherapy, chemotherapy, prognostic factors.

Contemp Oncol (Pozn) 2014; 18 (2): 116-119 DOI: $10.5114 /$ wo.2014.41392

\section{Metaplastic breast carcinomas - analysis of prognostic factors in a case series}

\author{
Elżbieta Nowara, Anna Drosik, Marzenna Samborska-Plewicka, \\ Ewa Magdalena Nowara, Agata Stanek-Widera
}

Maria Sklodowska-Curie Memorial Cancer Center and Institute of Oncology, Gliwice Branch, Poland

\section{Introduction}

Metaplastic breast carcinomas (MBC) are a rare and heterogeneous group of malignant breast cancers. In addition to infiltrating ductal cancer, MBC are characterized by the presence of other cellular types, typically squamous epithelial or mesenchymal components. The current WHO 2012 classification distinguishes 5 subtypes: low-grade adenosquamous carcinoma, fibromatosis-like metaplastic carcinoma, squamous cell carcinoma, spindle cell carcinoma, and carcinoma with mesenchymal differentiation (chondroid differentiation, osseous differentiation, and other types of mesenchymal differentiation).

Metaplastic breast carcinomas make up less than $1 \%$ of all breast cancers [1]. As they are very rare cancer types, their etiopathogenesis has not been fully elucidated. Distinctive clinical and pathological features of MBC include larger tumor size and less frequent metastases to the axillary lymph nodes at the time of diagnosis compared with typical ductal cancers [2-6]. Metaplastic breast carcinomas have so far been reported only in women. The expression of steroid receptors and overexpression of the HER2 receptor are identified in less than $25 \%$ of cases [5]. The majority of MBC have the characteristics of basal cancers, i.e. absence of steroid receptor expression or HER2 receptor overexpression, as well as demonstrating a high Ki-67 score [7]. Most literature reports have indicated that MBC are associated with a worse response to standard chemotherapeutic regimens and poorer patient prognosis than typical ductal carcinomas of the breast including "triple negative" cancers $[8,9]$. Since $M B C$ are very uncommon, there are no separate recommendations regarding therapy. Consequently, $\mathrm{MBC}$ management is the same as the treatment of ductal cancer. The standard regimen involves surgery combined with adjuvant therapy $[10,11]$. There are no definite evidence-based data on the efficacy of radiotherapy. In view of poor or absent expression of steroid receptors, hormone therapy is of marginal importance as a treatment option.

\section{Aim of the study}

The study is an outline of experiences with the treatment of patients diagnosed with $\mathrm{MBC}$ in one single institution. A review of the literature on the topic is also provided.

\section{Material and methods}

The study was performed at the Clinical and Experimental Oncology Department Cancer Center and Institute of Oncology Gliwice Branch (COI), which keeps an electronic database of patients allowing the selection of appropriate data for the purpose of this study. A retrospective analysis of medical records of MBC patients treated in the COI in 2006-2011 was performed. 
All the patients were diagnosed, treated and followed up at the COI. The authors had access to microscopic preparations, complete clinical data and information about consecutive follow-up examinations for all the study patients.

The analysis comprises 18 women. The median age of the patients at diagnosis was 63 years (range: 49-77 years). All the patients were in the menopause period.

Disease-free survival time was defined as time from the date of end of treatment until disease progression. Overall survival was defined as time from the date of diagnosis until death or the date of last contact. The survival was estimated by using the Kaplan-Meier method. A $p$ value less than 0.05 was considered as statistically significant. Statistica for Windows v.9.0 was used for statistical analysis.

\section{Results}

\section{Clinical diagnosis}

The median size of the primary tumor was $5 \mathrm{~cm}$ (range: 2-12 cm). Preliminary clinical examination revealed enlarged lymph nodes in the axilla in three patients (17\%). They were diagnosed with metaplastic carcinoma with squamous epithelial differentiation. One patient, who presented with an 8-month medical history, had a primary tumor measuring $12 \mathrm{~cm} \times 9 \mathrm{~cm}$. She was also diagnosed with primary metastases to the lungs and bones.

\section{Imaging tests}

Mammography and breast ultrasound were performed in all the patients. In the majority of women imaging tests revealed a poorly defined tumor with projections and microcalcifications. In only one patient mammography was negative. A detailed analysis of test findings revealed no characteristic signs of metaplastic carcinoma.

\section{Microscopic diagnosis}

Microscopic test results confirmed MBC with squamous epithelial differentiation in 10 patients (56\%). Sarcomatous differentiation was identified in four patients, chondral in three patients, and osseous in one. Postoper-

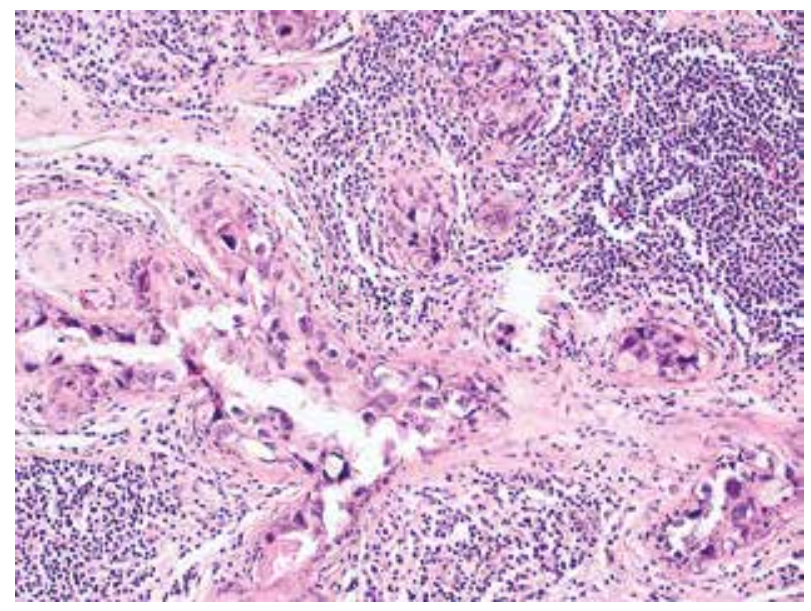

Fig. 1. Metaplastic breast carcinoma (low-grade adenosquamous carcinoma). Magnification 200x ative microscopic examination disclosed metastases to the axillary lymph nodes in four cases. All of them were revealed to have carcinoma with squamous epithelial differentiation (Fig. 1).

Only one patient was shown to have slight overexpression of the estrogen receptor. No steroid receptor or HER2 overexpression was found in the remaining patients.

\section{Treatment}

All the patients underwent surgical treatment. In one patient only simple mastectomy was performed due to advanced cancer stage. The remaining patients had radical mastectomy. One patient did not receive adjuvant chemotherapy and had surgery followed by individualized radiation therapy. The other patients were treated with chemotherapy, with three receiving neoadjuvant chemotherapy. All the patients were administered anthracyclines and half of them were additionally treated with 5 FU. The most common regimen was AC (cyclophosphamide, Adriamycin) and FAC (5 FU, Adriamycin, cyclophosphamide). The types of chemotherapy given were dependent on the type of healthcare funding. A total of 4-6 cycles were administered. Overall, 13 patients (72\%) had radiotherapy. Radical radiation doses were delivered, with the total dose amounting to 45-50 Gy in fractional doses of 2-2.5 Gy. Lack of steroid or HRE2 receptors makes these tumors extremely resistant to targeted therapy.

\section{Treatment outcomes}

All the patients had follow-up examinations at the COI according to the schedule for breast cancer patients.

The median follow-up period was 31.3 months. During that time six patients developed metastatic disease. Furthermore, in all of them there were metastases to the lung. Concurrent metastasis to the brain was confirmed in one woman. Local recurrence was detected in another three cases. There were no bone metastases. Cancer relapse was confirmed in $50 \%$ of the study patients, with a median time to relapse of 17.5 months. Five patients received cisplatin-based chemotherapy due to metastases or inoperable local recurrence. The disease progressed in

Table 1. Clinicopathological patient characteristics

\begin{tabular}{lcc}
\hline \multicolumn{1}{c}{ Feature } & $\begin{array}{c}\text { Number of } \\
\text { patients }\end{array}$ & (\%) \\
$\begin{array}{l}\text { tumor size }[\mathrm{cm}] \\
\text { range } 2-12 \\
\text { median } 5\end{array}$ & 4 & \\
nodal metastases & 10 & 22 \\
\hline squamous epithelial differentiation & 4 & 56 \\
\hline sarcomatous differentiation & 3 & 22 \\
chondral differentiation & 1 & 17 \\
osseous differentiation & 9 & 5 \\
\hline cancer relapse & 6 & 50 \\
\hline distant metastases & & 33
\end{tabular}




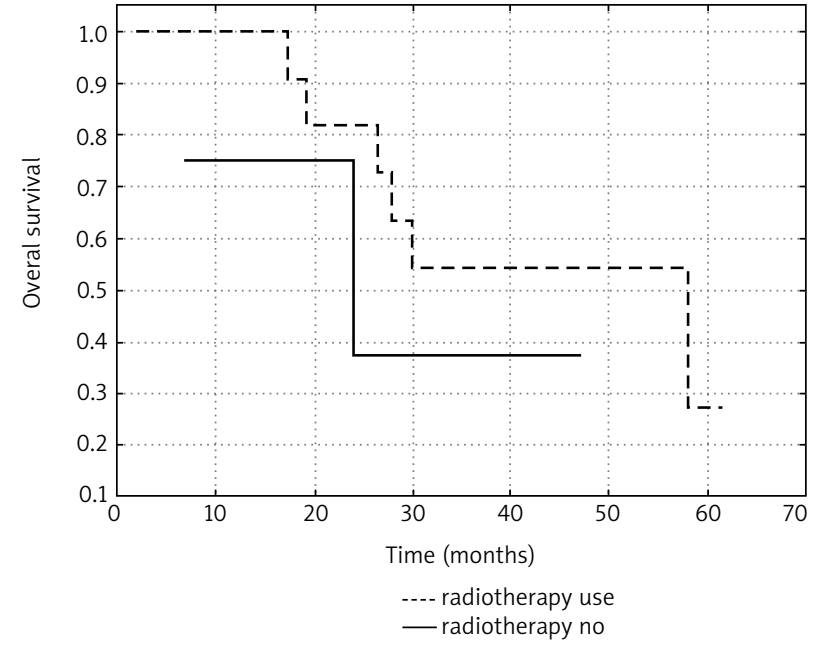

Fig. 2. Overall survival according to radiotherapy use

three patients during therapy, with one patient from this group still alive at the time of writing this article.

Nine patients died during the follow-up period. All the deaths were due to the progression of cancer. The median disease-free survival time was 6.5 months. A total of nine patients were alive as of the completion of the follow-up. Six of them had no evidence of cancer.

Clinicopathological patient characteristics are given in the Table 1.

\section{Prognostic factors}

A number of clinical factors (including age at diagnosis, tumor size, lymph node involvement, microscopic type of cancer, family history, number of childbirths) were assessed to determine their influence on survival. None of the factors listed above were found to have a significant effect on patient survival.

Radiation treated patients, however, were observed to live significantly longer in comparison with women who had no radiotherapy, 42 and 27 months respectively, $p=$ $=0.018$ (Fig. 2). Cisplatin therapy had no significant effect on overall survival, $p=0.17$.

\section{Discussion}

Analyses found in the medical literature rarely include more than 20 cases of MBC because of their rarity. In imaging tests metaplastic carcinomas do not exhibit any characteristic features aside from greater propensity for the formation of cystic lesions observed in carcinomas with the squamous epithelial component [6]. In the group of patients under study imaging tests showed no atypical signs. Metaplastic carcinomas rarely cause the expression of steroid or HER2 receptors (detected in only up to $25 \%$ of cases). In the group of patients enrolled in the present study estrogen receptor expression was identified in just one case, with no expression detected in the remaining patients. No HER2 overexpression was present in any of the patients, which corroborates the findings of other authors. Metaplastic breast carcinomas cells have a lower degree of estrogen and progesterone receptor expression, and HER2 expression, and higher Ki-67 and p-53 scores compared to ductal cancers [7, 12]. Genetic profiling shows deregulation of BRCA1, PTEN and TOP2A pathways (molecular targets of doxorubicin), which may account for the lower proclivity of MBC for forming metastases in the lymph nodes, resistance to chemotherapy and, possibly, sensitivity to radiotherapy [7].

There are reports of good therapeutic responses, including nearly complete pathological responses, to cisplatin-containing chemotherapy in metaplastic cancers with a squamous epithelial component [13]. Low incidence of $M B C$ in combination with scarce literature data, however, make it impossible to draw any definite conclusions about the optimum treatment regimen. In the group of patients reported here there were five cisplatin-treated patients. Disease progression during therapy was confirmed in three of them. The median primary tumor size in the study group analyzed here was $5 \mathrm{~cm}$. Metastases in the lymph nodes of the axillary fossa were detected in three cases (17\%). The authors report extensive involvement of the lymph nodes, ranging between 8 and $56 \%$ of cases, though the majority of authors indicate a low rate of metastasis to the axillary lymph nodes [15].

Patient prognosis is mainly determined by the extent of the metaplastic component and its type, a markedly worse prognosis being linked to tumors with a sarcomatous component [13]. No such trend was observed in our limited group of patients. The patients relapsed regardless of the microscopic subtype of cancer. Distant metastases are mainly located in the lungs [15]. Among the patients in the present study there were also distant metastases identified in the lungs. One patient developed primary metastases in the lungs and bones. Cancer relapse was confirmed in half of the patients, which is consistent with results obtained by other researchers. Some authors claim that despite a lower rate of lymph node metastasis patients suffering from MBC show shorter overall survival, which might be an argument prompting physicians to administer adjuvant radiation therapy more often [11]. In the group reported here, $72 \%$ of patients received radiotherapy. The indications were the same as in ductal cancers.

Most researchers believe that MBC is linked to worse prognosis, which suggests that further studies are necessary to investigate the disease in greater detail.

In conclusions metaplastic breast carcinomas are an uncommon cancer type. Longer overall survival time among the study patients was observed for women who underwent postoperative adjuvant radiation therapy, $p=0.018$. The most common location of metastases was the lungs. Lack of steroid or HER2 receptors makes these tumors extremely resistant to targeted therapy. Since the study group was small, the results should be approached with appropriate caution [17].

The authors declare no conflict of interest. 


\section{References}

1. Park JH, Han W, Kim SW, et al. The clinicopathologic characteristics of 38 metaplastic carcinomas of the breast. J Breast Cancer 2005 . 8: 59-63.

2. Beatty JD, Atwood M, Tickman R, Reiner M. Metaplastic breast cancer: clinical significance. Am J Surg 2006; 191: 657-64.

3. Chao TC, Wang CS, Chen SC, Chen MF. Metaplastic carcinomas of the breast. J Surg Oncol 1999; 71: 220-5.

4. Barnes PJ, Boutilier R, Chiasson D, Rayson D. Metaplastic breast carcinoma: clinical-pathologic characteristics and HER2/neu expression. Breast Cancer Res Treat 2005; 91: 173-8.

5. Pezzi CM, Patel-Parekh L, Cole K, Franko J, Klimberg VS, Bland K. Characteristics and treatment of metaplastic breast cancer: analysis of 892 cases from the National Cancer Data Base. Ann Surg Oncol 2007; 14: 166-73.

6. Park JM, Han BK, Moon WK, Choe YH, Ahn SH, Gong G. Metaplastic carcinoma of the breast: mammographic and sonographic findings. J Clin Ultrasound 2000; 28: 179-86.

7. Weigelt B, Kreike B, Reis-Filho JS. Metaplastic breast carcinomas are basal-like breast cancers: a genomic profiling analysis. Breast Cancer Res Treat 2009; 117: 273-80.

8. Chen IC, Lin CH, Huang CS, Lien HC, Hsu C, Kuo WH, Lu YS, Cheng AL. Lack of efficacy to systemic chemotherapy for treatment of metaplastic carcinoma of the breast in the modern era. Breast Cancer Res Treat 2011; 130: 345-51.

9. Lee H, Jung SY, Ro JY, et al. Metaplastic breast cancer: clinicopathological features and its prognosis. J Clin Pathol 2012; 65: 441-6.

10. Beatty JD, Atwood M, Tickman R, Reiner M. Metaplastic breast cancer: clinical significance. Am J Surg 2006; 191: 657-64.

11. Chao TC, Wang CS, Chen SC, Chen MF. Metaplastic carcinomas of the breast. J Surg Oncol 1999; 71: 220-5.

12. Tseng WH, Martinez SR. Metaplastic breast cancer: to radiate or not to radiate? Ann Surg Oncol 2011; 18: 94-103.

13. Takuwa H, Ueno T, Ishiguro H, Mikami Y, Kanao S, Takada M, Sugie T, Toi M. A case of metaplastic breast cancer that showed a good response to platinum-based preoperative chemotherapy. Breast Cancer 2011 Apr 28 [Epub ahead of print].

14. Behranwala KA, Nasiri N, Abdullah N, Trott PA, Gui GP. Squamous cell carcinoma of the breast: clinico-pathologic implications and outcome. Eur J Surg Oncol 2003; 29: 386-9.

15. Tse GM, Tan PH, Putti TC, Lui PC, Chaiwun B, Law BK. Metaplastic carcinoma of the breast: a clinicopathological review. J Clin Pathol 2006; 59: 1079-83.

16. Rayson D, Adjei AA, Suman VJ, Wold LE, Ingle JN. Metaplastic breast cancer: prognosis and response to systemic therapy. Ann Oncol 1999; 10: 413-9.

17. Wu YY, Yen MF, Yu CP, Chen HH. Individually tailored screening of breast cancer with genes, tumour phenotypes, clinical attributes, and conventional risk factors. Br J Cancer 2013; 108: 2241-2249.

\section{Address for correspondence}

Elżbieta Nowara MD, PhD

Maria Sklodowska-Curie Memorial Cancer Center and Institute of Oncology

Gliwice Branch

Wybrzeże AK 15

44-100 Gliwice, Poland

e-mail: enowara@aol.pl

Submitted: 21.05 .2013

Accepted: $\quad 6.08 .2013$ 